\title{
Surface effects on shape, self-organization and photoluminescence of InAs islands grown on InAIAs/InP(001)
}

\author{
J. Brault, ${ }^{a)}$ M. Gendry, ${ }^{\text {b) }}$ G. Grenet, and G. Hollinger \\ LEOM, UMR CNRS 5512, Ecole Centrale de Lyon, 36 avenue Guy de Collongue, \\ BP 16369131 Ecully Cedex, France \\ J. Olivares, B. Salem, T. Benyattou, and G. Bremond \\ LPM, UMR CNRS 5511, INSA de Lyon, Bât. 50269621 Villeurbanne Cedex, France
}

(Received 10 December 2001; accepted for publication 5 April 2002)

\begin{abstract}
InAs nanostructures were grown on $\operatorname{In}_{0.52} \mathrm{Al}_{0.48}$ As alloy lattice matched on $\operatorname{InP}(001)$ substrates by molecular beam epitaxy using specific growth parameters in order to improve island self-organization. We show how the change in InAs surface reconstruction via growth temperature from $(2 \times 4)$ to $(2 \times 1)$ and/or the use of InAlAs initial buffer surface treatments improve the island shape homogeneity (either as quantum wires or as quantum dots). Differences in island shape and in carrier confinement are shown by atomic force microscopy and by photoluminescence measurements, respectively. We point out that such shape amendments induce drastic improvements to island size distribution and discernible changes in photoluminescence properties, in particular concerning polarization. (C) 2002 American Institute of Physics. [DOI: 10.1063/1.1481959]
\end{abstract}

\section{INTRODUCTION}

In recent years, a considerable amount of work has been devoted to understanding and controlling the formation of self-assembled nanostructures by the Stranski-Krastanov growth mode, because this method today appears to be the most promising one to make quantum dots or quantum wires. For the highly mismatched system $(\mathrm{Ga}) \mathrm{InAs} / \mathrm{GaAs}^{1,2}$ (mismatch up to $7 \%$ ), it has led to novel quantum dot lasers ${ }^{3}$ and photodetectors. ${ }^{4,5}$ Likewise, the InAs/InP system (mismatch $\sim 3 \%$ ) has attracted considerable interest for laser sources at $1.55 \mu \mathrm{m} .{ }^{6,7}$ Moreover, because the lateral quantum confinement in InAs islands in InAlAs matrix lattice matched to an InP substrate ${ }^{8}$ allows huge normal-incidence intraband absorption, ${ }^{9}$ novel quantum dot infrared photodetectors can be realized. ${ }^{10}$ However, to fully take advantage of the optical and electronic properties of these low-dimensional structures, an accurate control of island size, shape, and distribution is needed in practice but this often presents a challenge. It has been shown that the morphological (and thus optical) properties of the islands critically depend on the growth conditions, ${ }^{11,12}$ on substrate orientation, ${ }^{13,14}$ and on the nature of the surrounding matrices (mainly because of alloying effects). ${ }^{15,16}$ For the particular InAs/InAlAs/InP(001) system we are concerned with in this article, the InAs island shape is somewhat unstable and both wire-like- and dot-like-shaped InAs islands can be observed ${ }^{12,17-19}$ deteriorating the morphological homogeneity and so the expected optical properties.

It is thus the aim of the present article to stress some growth parameters which are able to better the shape type

\footnotetext{
a) Present address: CEA-DRFMC-Service des Matériaux et Microstructures, 17 rue des Martyrs, 38054 Grenoble Cedex 9, France.

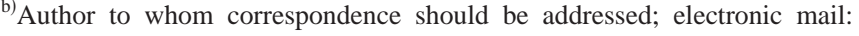
michel.gendry@ec-lyon.fr.
}

and size homogeneity of InAs islands when grown on InAlAs/InP(001) by conventional molecular beam epitaxy (MBE). In particular, we have identified two which are accountable for island shape: (i) the InAs surface reconstruction, i.e., $(2 \times 1)$ or $(2 \times 4)$, observed by reflection highenergy electron diffraction (RHEED) during twodimensional (2D) growth and (ii) the InAlAs buffer surface morphology previous to the InAs deposition.

\section{EXPERIMENTAL PROCEDURES}

The initial idea for this series of experiments was thus twofold: (i) to engineer the buffer surface during a growth interruption previous to the island growth, and (ii) to influence the island growth itself by varying the growth temperature and thus the surface reconstruction. Taking the expected connection between island morphology and photoluminescence (PL) properties into account, two series of samples were grown, namely, series I containing uncapped samples (referred as A-F) appropriate for atomic force microscopy (AFM) and series II containing similar but capped samples (referred as G-I) suitable for PL measurements. For the sake of clarity, the growth conditions for these two series are given separately in Tables I and II, respectively, along with the results from the characterization methods they have been designed for.

All the samples were grown by solid-source MBE on epi-ready semi-insulating $\operatorname{InP}(001)$ wafers from $\mathrm{InPact}$ in a Riber 2300 reactor. The substrate temperature was measured using an infrared pyrometer previously calibrated from the melting point $\left(525^{\circ} \mathrm{C}\right)$ of InSb. After thermal desorption at $565^{\circ} \mathrm{C}$ of the InP native oxide, a $400 \mathrm{~nm} \operatorname{In}_{0.52} \mathrm{Al}_{0.48} \mathrm{As}$ buffer layer, lattice matched with $\operatorname{InP}(001)$, was grown. Its growth temperature was fixed at $525^{\circ} \mathrm{C}$, the growth rate at 1 $\mu \mathrm{m} / \mathrm{h}$ and the V/III beam equivalent pressure (BEP) ratio at 20. At this point and before the InAs deposition, growth was 
TABLE I. RHEED and AFM results of InAs islands grown on InAlAs buffer layers on InP(001) substrates as a function of growth parameters. $\Delta d / d$ represents the mean island size dispersion obtained by calculating the average of the island's height, width, and length dispersions.

\begin{tabular}{|c|c|c|c|c|c|c|c|c|c|}
\hline \multirow[b]{2}{*}{ Sample } & \multirow{2}{*}{$\begin{array}{c}\text { InAlAs } \\
\text { Time spent } \\
\text { under As } \\
\text { (s) }\end{array}$} & \multicolumn{2}{|c|}{ InAs } & \multicolumn{6}{|c|}{ AFM } \\
\hline & & $\begin{array}{l}\text { Growth } \\
\text { temperature } \\
\left({ }^{\circ} \mathrm{C}\right)\end{array}$ & $\begin{array}{l}\text { RHEED } \\
\text { diagram }\end{array}$ & $\begin{array}{c}\text { Length } \\
(\mathrm{nm})\end{array}$ & $\begin{array}{c}\text { Width } \\
(\mathrm{nm})\end{array}$ & $\begin{array}{l}\text { Height } \\
(\mathrm{nm})\end{array}$ & $\begin{array}{l}\text { Island size } \\
\text { dispersion } \\
(\Delta d / d)(\%)\end{array}$ & Density & Shape \\
\hline A & 600 & 515 & $(2 \times 4)$ & $\begin{array}{l}170 \\
\pm 50\end{array}$ & $\begin{array}{c}36 \\
\pm 6.5\end{array}$ & $\begin{array}{c}1.9 \\
\pm 0.7\end{array}$ & 28 & $\begin{array}{l}\sim 300 / \\
\mu \mathrm{m}^{2}\end{array}$ & $\cdots$ \\
\hline B & 600 & 500 & $(2 \times 4)$ & $\cdots$ & $\begin{array}{c}25 \\
\pm 2.3\end{array}$ & $\begin{array}{c}1.7 \\
\pm 0.5\end{array}$ & 19 & $\begin{array}{l}\sim 35 / \\
\mu \mathrm{m}\end{array}$ & Wires \\
\hline $\mathrm{C}$ & 600 & 525 & $(2 \times 1)$ & $\begin{array}{c}56 \\
\pm 5.5\end{array}$ & $\begin{array}{c}27 \\
\pm 3.5\end{array}$ & $\begin{array}{c}1.5 \\
\pm 0.55\end{array}$ & 20 & $\begin{array}{l}\sim 650 / \\
\mu \mathrm{m}^{2}\end{array}$ & Dots \\
\hline $\mathrm{D}$ & $600^{\mathrm{a}}$ & 525 & $(2 \times 1)$ & $\begin{array}{l}52 \\
\pm 5\end{array}$ & $\begin{array}{c}29 \\
\pm 3.5\end{array}$ & $\begin{array}{c}1.6 \\
\pm 0.4\end{array}$ & 16 & $\begin{array}{l}\sim 800 / \\
\mu \mathrm{m}^{2}\end{array}$ & Dots \\
\hline $\mathrm{E}$ & 0 & 515 & $(2 \times 4)$ & $\ldots$ & $\begin{array}{c}14 \\
\pm 1.2\end{array}$ & $\begin{array}{c}1.4 \\
\pm 0.3\end{array}$ & 15 & $\begin{array}{l}\sim 55 / \\
\mu \mathrm{m}\end{array}$ & Wires \\
\hline $\mathrm{F}$ & 0 & 525 & $(2 \times 1)$ & $\cdots$ & $\begin{array}{l}24 \\
\pm 3\end{array}$ & $\begin{array}{c}1.8 \\
\pm 0.3\end{array}$ & 25 & $\begin{array}{l}\sim 35 / \\
\mu \mathrm{m}\end{array}$ & Wires \\
\hline
\end{tabular}

${ }^{\mathrm{a} P l u s}$ a few seconds under reduced As pressure.

interrupted for some samples, namely, samples A-D in series I, and samples H-I in series II. During this growth interruption, procedures we judged able to reduce the natural morphologic anisotropy of an As-stabilized InAlAs surface were carried out. Specifically, samples A-C were maintained under arsenic overpressure $\sim 4 \times 10^{-6}$ Torr for $600 \mathrm{~s}$. In addition to the previous treatment, sample D was left for a few seconds under reduced arsenic pressure, i.e., $2 \times 10^{-7}$ Torr, obtained by closing the main shutter. Turning now to samples designed for PL measurements, the buffer surface treatment for sample $\mathrm{G}$ was similar to that for sample $\mathrm{E}$ or $\mathrm{F}$ i.e., no growth interruption. The buffer surface treatments for samples I and D are alike (kept for $5 \mathrm{~s}$ under $2 \times 10^{-7}$ Torr As pressure), though for sample $H$ there is a slight difference with sample D: besides a growth interruption of $600 \mathrm{~s}$ under As pressure, sample $\mathrm{H}$ was kept for 20 s under 7 $\times 10^{-7}$ Torr As pressure.

After these buffer-surface engineering treatments, 3.5 ML equivalent thickness of InAs was grown on all samples. This equivalent thickness is just above the critical threshold for the 2D/three-dimensional (3D) growth mode transition (2.5 ML) as measured by RHEED. In each case, the InAs reduced growth rate was fixed at $0.25 \mu \mathrm{m} / \mathrm{h}(0.22 \mathrm{ML} / \mathrm{s})$ and the arsenic BEP was fixed around $4 \times 10^{-6}$ Torr to ensure As-rich growth conditions. Two different growth temperature ranges were chosen as the means to obtain a well-controlled
InAs RHEED reconstruction: $500-515^{\circ} \mathrm{C}$ for a $(2 \times 4)$ reconstruction (samples A, B, and $\mathrm{E}$ in series I and sample $\mathrm{G}$ in series II) and $520-525^{\circ} \mathrm{C}$ for a $(2 \times 1)$ reconstruction (samples C, D, and F in series I and samples $\mathrm{H}$ and I in series II).

After the InAs growth, the samples were held for $30 \mathrm{~s}-2$ min at growth temperature to stabilize the island shape. Uncapped samples $(\mathrm{A}-\mathrm{F})$ designed for AFM imaging were then rapidly cooled down to $300{ }^{\circ} \mathrm{C}$ (with arsenic pressure kept unchanged in order to reduce as far as possible the surface reorganization) and transferred into air to be imaged by $e x$ situ AFM. AFM imaging was made using a Park Scientific Instruments microscope, model $\mathrm{CP}$, operating in the contact mode. Furthermore, a $300 \mathrm{~nm}$ lattice-matched InAlAs cap layer was grown for the three extra samples designed for optical characterization. PL spectra were performed at 8 and $300 \mathrm{~K}$ within a closed cycle helium cryostat. The PL was excited using the $514.5 \mathrm{~nm}$ line of an argon-ion laser. The PL emission was dispersed by a high resolution spectrometer and measured by a high-sensitivity $77 \mathrm{~K}$ cooled germanium photodetector using a conventional lock-in technique.

\section{RESULTS AND DISCUSSION}

Typical AFM images of uncapped samples are shown in Fig. 1. As already mentioned, the corresponding island

TABLE II. PL results (at 300 and $8 \mathrm{~K}$ ) vs growth parameters and InAs island shape.

\begin{tabular}{|c|c|c|c|c|c|c|c|c|}
\hline \multirow[b]{3}{*}{ Sample } & \multirow{3}{*}{$\begin{array}{c}\text { InAlAs } \\
\text { Time spent } \\
\text { under As } \\
\text { (s) }\end{array}$} & \multicolumn{3}{|c|}{ InAs } & \multicolumn{4}{|c|}{ PL } \\
\hline & & \multirow{2}{*}{$\begin{array}{c}\text { Growth } \\
\text { temperature } \\
\left({ }^{\circ} \mathrm{C}\right)\end{array}$} & \multirow[b]{2}{*}{$\begin{array}{l}\text { RHEED } \\
\text { diagram }\end{array}$} & \multirow[b]{2}{*}{$\begin{array}{l}\text { Island } \\
\text { shape }\end{array}$} & \multicolumn{2}{|c|}{$300 \mathrm{~K}$} & \multicolumn{2}{|c|}{$8 \mathrm{~K}$} \\
\hline & & & & & $\begin{array}{c}E_{g} \\
(\mathrm{eV})\end{array}$ & $\begin{array}{c}\text { FWHM } \\
(\mathrm{meV})\end{array}$ & $\begin{array}{c}E_{g} \\
(\mathrm{eV})\end{array}$ & $\begin{array}{c}\text { FWHM } \\
(\mathrm{meV})\end{array}$ \\
\hline $\mathrm{G}$ & 0 & 515 & $(2 \times 4)$ & Wire & 0.92 & 128 & 1.07 & 132 \\
\hline $\mathrm{H}$ & $600^{\mathrm{a}}$ & 525 & $(2 \times 1)$ & Dot & 0.88 & 117 & 1.04 & 129 \\
\hline I & $600^{\mathrm{b}}$ & 525 & $(2 \times 1)$ & Dot & 0.92 & 118 & 1.11 & 159 \\
\hline
\end{tabular}

aplus $20 \mathrm{~s}$ under $7 \times 10^{-7}$ Torr.

bPlus 5 s under $2 \times 10^{-7}$ Torr. 

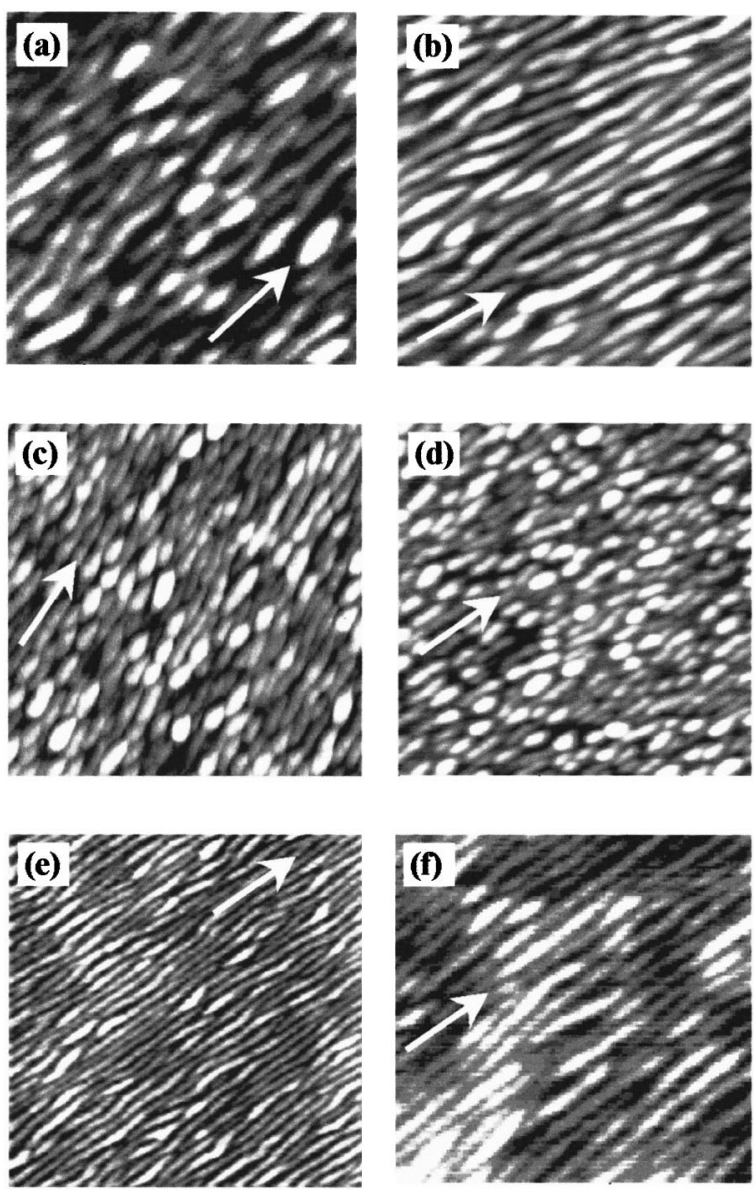

\section{$\overline{0.2 \mu \mathrm{m}}$}

FIG. 1. Ex situ AFM images $\left(0.5 \times 0.5 \mu \mathrm{m}^{2}\right)$ of InAs island morphology for sample A (a), sample B (b), sample C (c), sample D (d), sample E (e), and sample $F(f)$. The white arrow indicates the [1 10$]$ direction.

growth conditions and AFM characteristics are collected in Table I. Whatever the growth conditions are, InAs islands always present a flat shape, i.e., average heights $(H)$ in the $0.8-1.9 \mathrm{~nm}$ range for average widths $(W)$ in the $14-36 \mathrm{~nm}$ range, that is to say, a ratio of at least $1-10$. The reference sample is sample A [Fig. 1(a)] obtained with nonoptimized growth conditions. Its surface shows: (i) a strong anisotropy of the island shapes which are elongated along the [1 10$]$ direction and (ii) an island alignment along the same [1 10$]$ direction which reinforces the overall wire-like aspect. Such elongation and alignment are observable for all samples in Fig. 1, but can be drastically reduced or enhanced by varying the InAs surface reconstruction regulated by the growth temperature and/or the buffer surface morphology controlled by growth interruptions. In short, the best wire-like shape [Fig. $1(\mathrm{e})]$ is obtained with no growth interruption at all and with an island growth at $500^{\circ} \mathrm{C}$ corresponding to an optimized $(2 \times 4)$ reconstructed InAs surface (sample E) while the best dot-like shape [Fig. 1(d)] is obtained with an optimized growth interruption and for a special $(2 \times 1)$ reconstructed InAs surface, viz, a strongly As depleted surface at the lower limit of the As stabilization at $525^{\circ} \mathrm{C}$ (Sample D).
Concerning the effect of InAs surface reconstruction, two types of island shape can be produced. Each of them has been related to the reconstruction, either a $(2 \times 4)$ or a $(2$ $\times 1$ ), obtained mainly according to the growth temperature but also to the arsenic pressure during the InAs island growth. For the arsenic pressures used in this study, we have found that temperatures between 500 and $515{ }^{\circ} \mathrm{C}$ yield a $(2$ $\times 4)$ reconstruction while higher temperatures yield a (2 $\times 1)$ reconstruction. When comparing Figs. 1(b) and 1(c), it can be observed that a $(2 \times 1)$ reconstruction induces a dotlike shape propensity while a wire shape is favored by a ( 2 $\times 4)$ reconstruction. We believe that the reason for such behavior can be found in the intrinsic InAs surface roughness anisotropy. It is now well established that nonstrained III-V material surface presents an intrinsic anisotropic surface roughness when As stabilized. This anisotropy has been interpreted either in terms of surface step energy formation (A and $\mathrm{B}$ steps are chemically different) or in terms of adatom mobility along the A or B surface step. ${ }^{20}$ Such surface anisotropy is thought to remain during the compressively strained 2D growth mode, since it is mainly due to the peculiar zinc blende bulk atomic structure which determines the A and B surface step direction and chemistry. When the 2D-3D transition occurs, there is some memory of the previous 2D growth mode (especially if the 2D-3D threshold is smooth) because initial steps are where elastic relaxation can take place. This memory will in turn induce the observed anisotropy for the ensuing 3D islands. ${ }^{21}$ However, this A and $B$ step nonequivalence can be either enhanced or reduced by an enhancement or a reduction of the percentage of As present at the surface, a percentage which can be directly monitored by RHEED reconstruction during the MBE growth. In our case, the most stable reconstruction is the well known $(2 \times 4)$ which corresponds roughly to $75 \%$ As coverage. This reconstruction is known to give rise to considerable surface anisotropy and, thus, a well-defined wire-like shape for the InAs islands is expected. If the growth temperature is increased, we think that the surface is As depleted compared to the previous $(2 \times 4)$ reconstructed one. This surface is, therefore, viewed by RHEED as a $(2 \times 1)$ reconstruction. In this case, the surface anisotropy being less pronounced, the formation of more isotropic InAs islands is predicted. Our results are in full agreement with the above argument as the best dots (sample D) are obtained for a strongly As depleted surface while the best wires (sample E) are obtained at low temperature for an optimized $(2 \times 4)$ As-rich surface.

Turning now to the influence of the InAlAs buffer surface morphology on the InAs island shape, a wire-like shape is obtained [Figs. 1(e) and 1(f)] when growth has not been interrupted between the InAlAs buffer and InAs layer growths while, on the contrary, a dot-like shape is observed [Figs. 1(c) and 1(d)] when the growth process is interrupted. The reason for this buffer effect on island shape can be attributed to an evolution of the buffer surface, both in morphology and composition, during the growth interruption. Specifically, this InAlAs surface roughening is well indicated by the RHEED pattern becoming more and more spotty during the growth interruption. In fact, during a long enough growth interruption, there is no kinetic limitation preventing 
the surface atoms from reaching optimal distribution on a surface that minimizes the total elastic plus surface energy. In the case of an InAlAs buffer, an immiscible alloy with a strongly positive mixing parameter (around $3600 \mathrm{cal} / \mathrm{mol}$ ), the growth interruption will favor a development of phase separation in order to reduce the intrinsic alloy strain energy. However, such a phase separation has been shown to be energetically favorable for an alloy which is lattice matched on a substrate only if its surface presents some roughness. ${ }^{22}$ In other words, an efficient way to partly relax the intrinsic alloy strain energy is by alloy demixing along with a surface roughening even if the surface energy concurrently increases as the surface area increases. Moreover, the surface energy itself depends on the propensity of alloys to lower their surface energy by segregating the most adequate binary towards the surface. In the present case, the InAs binary has a lesser surface energy than the AlAs binary and thus will segregate on the surface. This process, being a short-range one, is less subject to anisotropy effects than the long-range growth process. To summarize, the main growth interruption effect on the surface of the InAlAs buffer is roughening due to surface alloy demixing, leading to InAs-rich, more or less isotropic humps acting as precursors for the subsequent InAs island growth.

To go farther, if the two effects discussed above (InAs surface reconstruction and InAlAs buffer surface preparation) are combined, drastic improvements can be obtained in island shape homogeneity and distribution, either for dots [Fig. 1(d)] or for wires (Fig. 1(e)]. Note that for sample D, a few extra seconds were spent under reduced As pressure after the $600 \mathrm{~s}$ growth interruption to gain even more isotropy. All in all, such control has led to shape size, and surface distribution improvement as can be seen from Table I: in particular, the $\Delta d / d$ distribution that quantifies the mean island-size dispersion (the average of the island height, width, and length dispersions) reduces from $28 \%$ for sample A to $16 \%$ and $15 \%$ for sample D (dots) and sample E (wires), respectively.

To complete this study, PL measurements were made at 300 and $8 \mathrm{~K}$ on InAlAs capped samples containing wire-like shape InAs islands (sample G similar to sample E) and dotlike shaped InAs islands (samples $\mathrm{H}$ and I similar to sample D). Growth conditions and PL results for these samples are given in Table II and Fig. 2. In all cases, PL peaks appear at almost the same energy around $1.07 \mathrm{eV}$ at $8 \mathrm{~K}$ for both dots and wires (Fig. 2). This is not surprising because dots and wires have almost the same heights (see Table I). As a matter of fact, the absolute peak position of PL peaks is expected to be predominantly determined by the island height (a lot lower than the island width) because confinement is stronger in the direction perpendicular to the island plane than in directions parallel to the island plane. Likewise, PL peak broadening is closely related to the height distribution of the islands and thus almost independent of the island shape. The result is that the full width at half maximum (FWHM) of the PL peaks at $8 \mathrm{~K}$ is also nearly the same for both dots and wires $(\sim 130 \mathrm{meV}$ for samples $\mathrm{G}$ and $\mathrm{H})$ but significantly smaller than the value we previously found ${ }^{9,16}$ for a nonoptimized island growth $(\sim 175 \mathrm{meV})$. More interesting is the

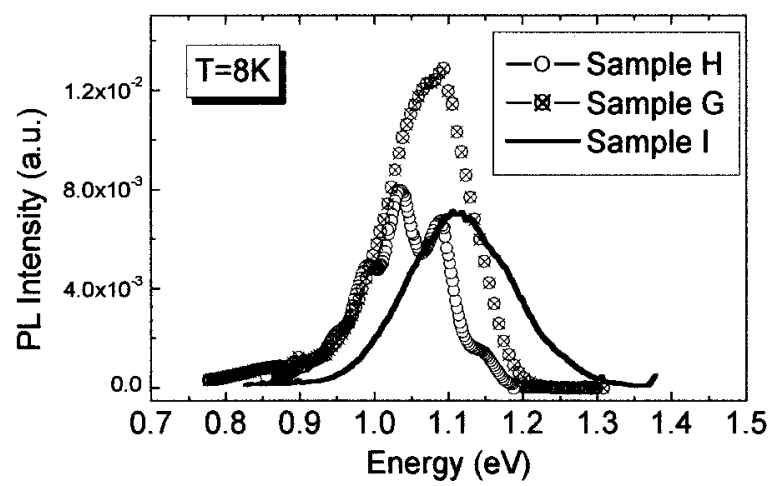

FIG. 2. Photoluminescence spectra at $8 \mathrm{~K}$ of InAs/InAlAs islands for sample G (InAs wires), samples H and I (InAs elongated dots).

shape difference between the PL spectra of sample G (with an unresolved broad form) and of sample $\mathrm{H}$ (revealing several resolvable components). As previously discussed, ${ }^{8,9,16}$ the $\mathrm{H}$-sample components are thought to arise from fundamental and excited states of dots having monolayer-height fluctuations. For sample G, we think that this effect cannot be seen because of a worse lateral confinement than in sample $H$ due to the island's wire shape. The origin of the odd unresolved PL spectrum for sample I is not yet well understood. It is worth noting that the overall integrated intensity measured at $300 \mathrm{~K}$ is only $2-4$ times lower than at 8 $\mathrm{K}$, confirming well carrier capture and spatial confinement in the InAs islands, the most efficient carrier spatial confinement being, as expected, for the dot-like islands. Finally, in order to specify the carrier confinement anisotropy associated with island shape anisotropy, polarized PL measurements (along the [110] and [1 $1 \overline{1} 0]$ directions) were carried out at $300 \mathrm{~K}$ to determine the linear polarization degree [defined as $([1 \overline{1} 0]-[110]) /[(1 \overline{1} 0]+[110]]$ of the emitted light. The curves are shown in Fig. 3 for sample $G$ (wires) and I (dots). For both the G and I cases (containing wires and dots, respectively), the PL intensity emission is stronger in the [1 10$]$ direction than in the [110] direction, in agreement with what is expected for the island elongation found by AFM measurements. However, there is a factor of nearly 2 between the linear polarization degrees of PL emissions of sample G (wires) and of sample I (dots), (around 30\% and $17 \%$, respectively).

The polarization degree of photoluminescence is sensitive to many factors. ${ }^{23-25}$ Among them, let us first cite the intrinsic anisotropy of the material used either for the matrix or the dots, especially if it is an alloy displaying strong phase separation or ordering. Second, since the shape of the dots is not truly cubic, the strain distribution and, consequently, the electronic energy eigenvalues and wave functions can strongly be dependent on this dot shape anisotropy. This clearly means some more anisotropy for the photoluminescence transitions. Moreover, we should take into account that the valence band is $p$ type and thus not spherical as the conduction $s$ band is. It results that here again, the photoluminescence polarization can be affected. It is quite difficult to go ahead without full theoretical calculations because photoluminescence intensities are well known to be extremely 

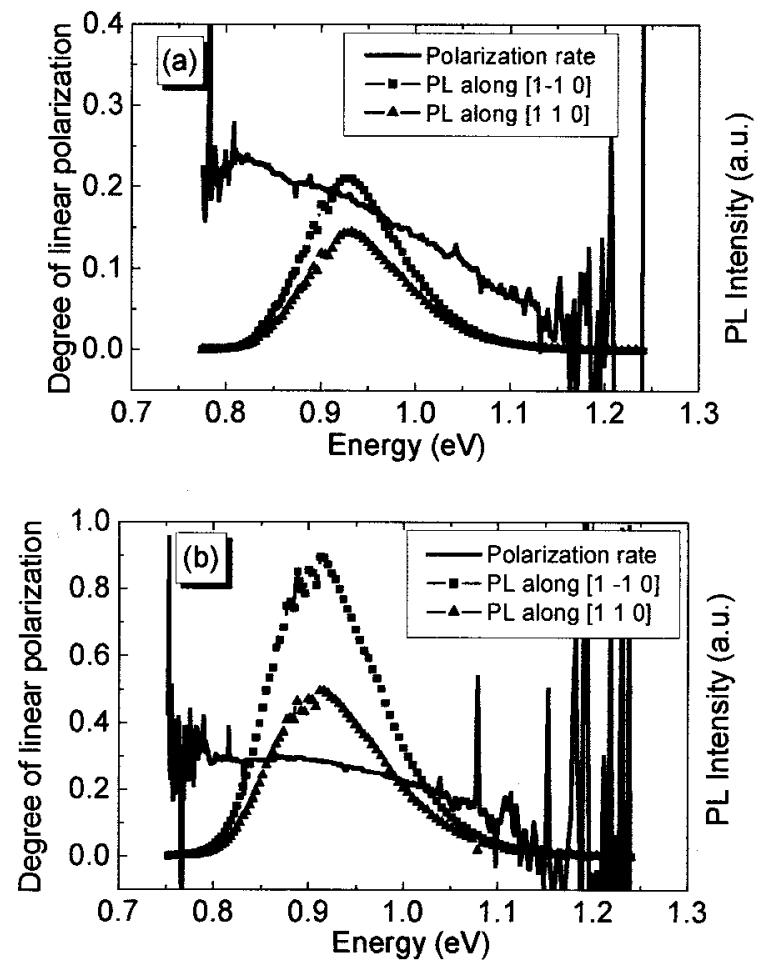

FIG. 3. Polarized photoluminescence spectra at $300 \mathrm{~K}$ along the [110] and [1 $1 \overline{1} 0]$ directions for: (a) sample I (InAs elongated dots) and (b) sample G (InAs wires).

more responsive than energies to strain field distribution or band interactions. To a large extent, to fully discuss this theoretical point is beyond the scope of this article. Let us just state that the InAlAs barrier is visibly a rather anisotropic material (80\% measured) and thus can bring some anisotropy even into the photoluminescence of a dot-like-shaped island. However, the shape effect still remains in our case a major factor since, for the same barrier, the anisotropy is found to be $30 \%$ for sample $\mathrm{G}$ (wires) to be compared with $17 \%$ found for sample I (dots).

\section{CONCLUDING REMARKS}

In summary, the shape of self-organized InAs islands grown on InAlAs/InP(001) appears to be strongly dependent on growth parameters, showing either a wire-like shape or a dot-like shape. The wire-like shape is enhanced if the InAs islands are grown with a well defined $(2 \times 4)$ reconstruction (in our case obtained with a growth temperature in the 500$515^{\circ} \mathrm{C}$ range) and without any growth interruption between the InAlAs-buffer and the InAs-island growths. In contrast, the dot-like shape is favored by an As-depleted $(2 \times 1)$ reconstruction (in our case with a growth temperature in the $520-525^{\circ} \mathrm{C}$ range) during the InAs growth associated with quite a long growth interruption (600 s) between the InAlAs buffer and the InAs growths. The reconstruction effect was attributed to the difference between A- and B-step energy for an As-stabilized surface as a function of As percentage, while the growth interruption effect was related to compositional and morphological evolutions of the InAlAs buffer surface, leading to (more isotropic than on the growth front) InAsrich regions, acting as precursors for the subsequent InAs island growth. Of perhaps more use to actual applications is that these islands with a better defined shape present a better self-organization in terms of size and spatial distribution, yielding quite a good carrier confinement as confirmed by PL measurements.

\section{ACKNOWLEDGMENTS}

The authors are grateful to M.-P. Besland for helpful discussions about AFM imaging. The authors would also like to thank J.-B. Goure for technical assistance. This work was supported by the Region Rhône-Alpes under Contract No. R0 1599/70.

${ }^{1}$ G. S. Solomon, J. A. Trezza, and J. S. Harris, Jr., Appl. Phys. Lett. 66, 991 (1995); 66, 3161 (1995)

${ }^{2}$ R. Leon, C. Lobo, A. Clark, R. Bozek, A. Wysmolek, A. Kurpiewski, and M. Kaminska, J. Appl. Phys. 84, 248 (1998).

${ }^{3}$ N. N. Ledentsov, V. A. Shchukin, M. Grundmann, N. Kirstaedter, J. Böhrer, O. Schmidt, D. Bimberg, V. M. Ustinov, A. Y. Egorov, A. E. Zhukov, P. S. Kop'ev, S. V. Zaitsev, N. Y. Gordeev, Z. I. Alferov, A. I. Borovkov, A. O. Kosogov, S. S. Ruvimov, P. Werner, U. Gösele, and J. Heydenreich, Phys. Rev. B 54, 8743 (1996).

${ }^{4}$ J. Phillips, K. Kamath, and P. Bhattacharya, Appl. Phys. Lett. 72, 2020 (1998).

${ }^{5}$ D. Pan, E. Towe, and S. Kennerly, Appl. Phys. Lett. 73, 1937 (1998).

${ }^{6}$ B. Lambert, A. Le Corre, V. Drouot, H. L'Haridon, and S. Loualiche, Semicond. Sci. Technol. 13, 143 (1998).

${ }^{7}$ N. Carlsson, T. Junno, L. Montelius, M.-E. Pistol, L. Samuelson, and W. Seifert, J. Cryst. Growth 191, 347 (1998).

${ }^{8}$ J. Brault, M. Gendry, G. Grenet, G. Hollinger, Y. Desières, and T. Benyattou, J. Cryst. Growth 201/202, 1176 (1999).

${ }^{9}$ A. Weber, O. Gauthier-Lafaye, F. H. Julien, J. Brault, M. Gendry, Y. Desières, and T. Benyattou, Appl. Phys. Lett. 74, 413 (1999).

${ }^{10}$ E. Finkman, S. Maimon, V. Immer, G. Bahir, S. E. Schacham, F. Fossard, F. H. Julien, J. Brault, and M. Gendry, Phys. Rev. B 63, 045323 (2001).

${ }^{11}$ L. González, J. M. García, R. García, F. Briones, J. Martínez-Pastor, and C. Ballesteros, Appl. Phys. Lett. 76, 1104 (2000).

${ }^{12}$ Y. F. Li, F. Lin, B. Xu, F. Q. Liu, X. L. Ye, D. Ding, and Z. G. Wang, J. Cryst. Growth 223, 518 (2001).

${ }^{13}$ R. Nötzel, J. Temmyo, A. Kozen, and T. Tamamura, Appl. Phys. Lett. 66, 2525 (1995)

${ }^{14}$ S. Fréchengues, N. Bertru, V. Drouot, B. Lambert, S. Robinet, S. Loualiche, D. Lacombe, and A. Ponchet, Appl. Phys. Lett. 74, 3356 (1999).

${ }^{15}$ V. M. Ustinov, E. R. Weber, S. Ruvimov, Z. Liliental-Weber, A. E. Zhukov, A. Yu. Egorov, A. R. Kovsh, A. F. Tsatsul'nikov, and P. S. Kop'ev, Appl. Phys. Lett. 72, 362 (1998).

${ }^{16}$ J. Brault, M. Gendry, G. Grenet, G. Hollinger, Y. Desières, and T. Benyattou, Appl. Phys. Lett. 73, 2932 (1998).

${ }^{17}$ S. Fafard, Z. Wasilewski, J. McLaffrey, and S. Raymond, Appl. Phys. Lett. 68, 991 (1996).

${ }^{18} \mathrm{H}$. Li, Q. Zhuang, X. Kong, Z. Wang, and T. Daniels-Race, J. Cryst. Growth 205, 613 (1999).

${ }^{19}$ J. Brault, M. Gendry, O. Marty, M. Pitaval, J. Olvares, G. Grenet, and G. Hollinger, Appl. Surf. Sci. 162/163, 584 (2000).

${ }^{20}$ S. B. Zhang and A. Zunger, Phys. Rev. B 53, 1343 (1996).

${ }^{21}$ A. Ponchet, A. Le Corre, A. Godefroy, S. Salaün, and A. Poudoulec, Microelectron. J. 26, 783 (1995).

${ }^{22}$ C. Priester and G. Grenet, J. Vac. Sci. Technol. B 16, 2421 (1998); Phys. Rev. B 61, 16030 (2000).

${ }^{23}$ M. Henini, S. Sanguinetti, S. C. Fortina, E. Grilli, M. Guzzi, G. Panzarini, L. C. Andreani, M. D. Upward, P. Moriarty, P. H. Beton, and L. Eaves, Phys. Rev. B 57, R6815 (1998).

${ }^{24}$ M. Sugisaki, H. W. Ren, S. V. Nair, K. Nishi, S. Sugou, T. Okuno, and Y. Masumoto, Phys. Rev. B 59, R5300 (1998).

${ }^{25}$ M. Notomi, M. Okamoto, H. Iwamura, and T. Tamamura, Appl. Phys. Lett. 62, 1094 (1993). 